TRABAJOS ORIGINALES

\title{
El género Attalea (Arecaceae) de Bolivia: Afinidades con sistemas ecológicos regionales
}

\author{
The genus Attalea (Arecaceae) of Bolivia: regional ecologic system affinities
}

\section{Mónica Moraes R. ${ }^{1 *}$ y Freddy S. Zenteno-Ruiz ${ }^{1,2}$}

\author{
1 Herbario Nacional de Bolivia, Instituto de Ecología, Universidad Mayor de San Andrés, Casilla 10077 - Correo Central, La Paz, Bolivia. \\ 2 Proyecto Identidad Madidi, WCS-Bolivia, La Paz, Bolivia, \\ *Autora de correspondencia \\ Email Mónica Moraes: monicamoraes45@gmail.com,mmoraes@fcpn.edu.bo \\ Email Freddy S. Zenteno-Ruiz: fred6zenruiz@gmail.com
}

\begin{abstract}
Resumen
La documentación de las especies neotropicales de la familia Arecaceae, basada en los recientes aportes a su taxonomía y su relación con los paisajes naturales, actualiza los patrones espaciales a los cuales se adaptan en su rango de distribución. En este caso se relevan 121 registros de especímenes de las 11 especies del género Attalea de Bolivia y su relación con 30 sistemas ecológicos que aproximan su ámbito de distribución a nivel regional. Para ello se sistematizó, se verificó y corrigieron las coordenadas geográficas vs. localidades de todos los especímenes coleccionados del género Attalea con el fin de cotejarlos con los sistemas ecológicos, utilizando las herramientas del ArgGis. Seguidamente elaboramos un dendrograma (especies vs. sistemas ecológicos) utilizando el método de distancia mínima en el programa R. El análisis de la relación de las especies con los sistemas ecológicos resalta una especie que no compone al sudoeste amazónico: $A$. eichleri y que procede de sistemas ecológicos del Cerrado. Entre las especies de Attalea amazónicas, $A$. blepharopus (endémica de Bolivia) se aísla de las demás y el resto subagrupa a especies según su presencia afín en bosques y sabanas, además del subandino y aluvial, como es para $A$. princeps, que se encuentra en 17 sistemas (57\%). Ocho especies de Attalea son comunes con Perú y 10 con Brasil. Es importante relacionar la agrupación jerárquica de las especies de Attalea con los sistemas ecológicos en función a dinámicas paisajísticas para documentar sus patrones de espacio y también para su conservación.
\end{abstract}

Palabras clave: Arecaceae; Attalea; Bolivia; dendrograma; sistemas ecológicos regionales.

\section{Abstract}

The documentation of the Neotropical species of the Arecaceae family, based on the recent contributions to its taxonomy and its relationship with natural landscapes, updates the spatial patterns to which they adapt in their range of distribution. In this case 121 records of specimens of the 11 species of the genus Attalea of Bolivia and their relationship with 30 ecological systems that approximate their scope of distribution at regional level are released. To this end, the geographical coordinates were systematized, verified and corrected. Localities of all the specimens collected from the genus Attalea in order to compare them with ecological systems, using the ArgGis tools. We then elaborate a dendrogram (species vs. ecological systems) using the minimum distance method in the R program. The analysis of the relation of the species with the ecological systems highlights a species that does not compose to the southwest amazon: $A$. eichleri and that is native to ecological systems of the Cerrado. Among the SW Amazonian Attalea species, A. blepharopus (endemic to Bolivia) is isolated from the others and the rest subgroup species according to their presence in forests and savannas, in addition to the subandean and alluvial, as it is for A. princeps, which is found in 17 systems (57\%). Eight species of Attalea are common with Peru and 10 with Brazil. It is important to relate the hierarchical grouping of the Attalea species with ecological systems in function of landscape dynamics to document their space patterns and also for their conservation.

Key words: Arecaceae; Attalea; Bolivia; dendrogram; regional ecological systems.

Citación:

Moraes R., M. \& F.S. Zenteno-Ruiz. 2017. El género Attalea (Arecaceae) de Bolivia: Afinidades con sistemas ecológicos regionales. Revista peruana de biología 24(3): 273 - 282 (octubre 2017). doi: http://dx.doi. org/10.15381/rpb.v24i3.13913

$\begin{array}{ll}\text { Presentado: } & 05 / 05 / 2017 \\ \text { Aceptado: } & 26 / 09 / 2017 \\ \text { Publicado online: } & 28 / 10 / 2017\end{array}$

Fuentes de financiamiento: El presente trabajo fue realizado con fondos del Proyecto Palmeras de Bolivia de la Universidad Mayor de San Andrés.

Información sobre los autores: MMR analizó los datos de distribución de las especies; FSZR utilizó las herramientas de GIS y la rectificación geográfica; ambos revisaron y aprobaron el manuscrito.

Los autores no incurren en conflictos de intereses. 


\section{Introducción}

Pese a los inventarios y relevamientos realizados en los últimos 10 años para documentar las palmeras sudamericanas, el género Attalea permanece todavía poco conocido (Pintaud 2008, Noblick et al. 2013, Pintaud et al. 2016). La mayor división en el género es geográfica y se inició hace 20 - 10 (promedio: 13) millones de años en el Mioceno como resultado de la separación de floras amazónicas y del Cerrado-Mata Atlántica, por lo que es considerado un género monofilético de reciente divergencia entre los demás Attaleinae con dispersiones hacia la Amazonia, Centro América y el Caribe (Meerow et al. 2009). Por su posición geográfica y latitudinal, en Bolivia se encuentran representadas especies de ambos clados considerando las afinidades fitogeográficas de la Amazonia, Andes, Cerrado y Gran Chaco (Tabla 1, Figura 1). Pintaud et al. (2008) mencionaron una lista total de 56 especies de Attalea, mientras que entre Colombia, Ecuador, Perú y Bolivia son 28 (Pintaud et al. 2015). Según Pintaud et al. (2008), el género Attalea es mucho más diverso en las formaciones periféricas secas que dentro de la misma Amazonía.

En un aporte reciente sobre las especies del sudoeste amazónico (que en adelante será mencionado como SWA, por las siglas en inglés: South Western Amazon) - que particularmente incluye a Perú, Bolivia y Brasil - se ańadieron 11 registros (Moraes \& Pintaud 2016a, Pintaud et al. 2016) y una nueva especie (Moraes \& Pintaud 2016b). Para el SWA son menos de 30 especies (Pintaud et al. 2015). En el caso de Bolivia, Moraes (2004) consideró un total de seis especies que a la fecha se incrementaron a 11 (Moraes 2015), fundamentalmente debido a un sesgo largamente no atendido en los herbarios de la región. Por ejemplo, en el caso de Attalea butyracea fue siempre citado con base a Henderson et al. (1995) donde se refiere una amplia distribución geográfica y se había incluido una amplia variedad de morfologías, hábitos y tipos de frutos. Ahora se ha constatado que esta especie está circunscrita a Colombia, Ecuador y Venezuela, por lo que la región del SWA se escindió en 13 nombres distintos del género Attalea (Pintaud et al. 2016). Además una especie considerada ampliamente distribuida y conocida en Bolivia como motacú, fue asignada a una especie: $A$. phalerata. Sin embargo, actualmente se ha circunscrito a $A$. princeps, teniendo ambas una extensa cobertura en el país.
Algunas de las especies de Attalea representan una importante fuente de recursos y materia prima para diferentes propósitos que benefician a las poblaciones locales. La categoría de uso que asigna mayor valor de uso es el de los frutos comestibles y la extracción de aceites de las semillas, así como el uso de las hojas para diferentes utensilios y artesanías, entre otros (Moraes 2014). En Bolivia se destacan dos especies: A. princeps Mart. (Paniagua-Zambrana \& Moraes, 2009) y A. speciosa Mart. (Toledo et al., 1999).

Como puntualizaron Pintaud (2008) y Meerow et al. (2009), la importante variación morfológica del género Attalea, en su sentido más amplio, va acompañada por la conformación de dos clados bien definidos: uno que incluye todas las especies del centro y este de Brasil (que corresponderían a lo que se conoció como Attalea sensu stricto) y el otro que abarca el resto de las especies como Attalea sensu lato. Sin embargo, A. tessmannii $\mathrm{Bu}-$ rret - que más bien no corresponde geográficamente al centro de Brasil - ha sido considerada como atípica en el antiguo concepto de Attalea sensu stricto (Rodríguez et al. 2016).

En esta contribución se plantean los siguientes objetivos: 1) Documentar las especies de Attalea que caracterizan diferentes paisajes naturales y 2) relacionar su presencia con sistemas ecológicos representados en la región SWA.

\section{Material y métodos}

Con un total de 121 colecciones de Attalea en Bolivia derivadas de bases de datos del Herbario Nacional de Bolivia (LPB), Herbario del Oriente Boliviano (USZ) y Herbario Forestal Martín Cárdenas (BOLV) se inició el registro del total de especies del género. Se consideró la lista preliminar de Arecaceae de Bolivia (Moraes 2015), la clave taxonómica y apuntes sobre las especies de Attalea compartidas por Bolivia, Perú y Brasil (Pintaud et al. 2016) respecto a las especies del SWA, así como la epitipificación de $A$. blepharopus Mart. (Moraes \& Pintaud 2016a) y la nueva especie descrita para esta región: $A$. pacencis Moraes y Pintaud (Moraes \& Pintaud 2016b).

Para la relación con los paisajes regionales se tomó en cuenta la clasificación de Josse et al. (2009), el mapa a detalle de los sistemas ecológicos compartidos entre Bolivia y Perú (Young 2007) y el correspondiente para Bolivia (Navarro \& Ferreira 2011).

Tabla 1. Relación fitogeográfica de especies de Attalea reportadas para Bolivia (adaptado según Moraes et al. 2014, Moraes 2015 y Velásquez-Puentes \& Bacon 2016) y formaciones de vegetación.

\begin{tabular}{lll}
\hline Especies de Attalea & Afinidad fitogeográfica & Formaciones de vegetación \\
\hline Attalea bassleriana & Amazonia & Bosque amazónico alto y ribereño en terrazas libres de inundación \\
Attalea blepharopus & Amazonia & Bosque subandino, muy húmedo \\
Attalea eichleri & Cerrado & Bosque semideciduo en sustratos rocosos \\
Attalea maripa & Amazonia & Bosque amazónico abierto y bordes de bosques secundarios \\
Attalea moorei & SW Amazonia & Bosque amazónico y subandino de tierras bajas \\
Attalea pacensis & SW Amazonia & Bosque amazónico y subandino de tierras bajas \\
Attalea peruviana & SW Amazonia & Bosque amazónico alto y ribereño \\
Attalea phalerata & Cerrado - Pantanal & Bordes de bosque amazónico, islas de bosque, bosques inundados \\
Attalea princeps & Amazonia & Bosques amazónicos y subandinos (hasta 1000 m de altitud) \\
Attalea speciosa & Amazonia - Cerrado & Bosques semideciduos y praderas abiertas en sustratos rocosos \\
Attalea tessmannii & SW Amazonia & Bosque amazónico alto \\
\hline
\end{tabular}



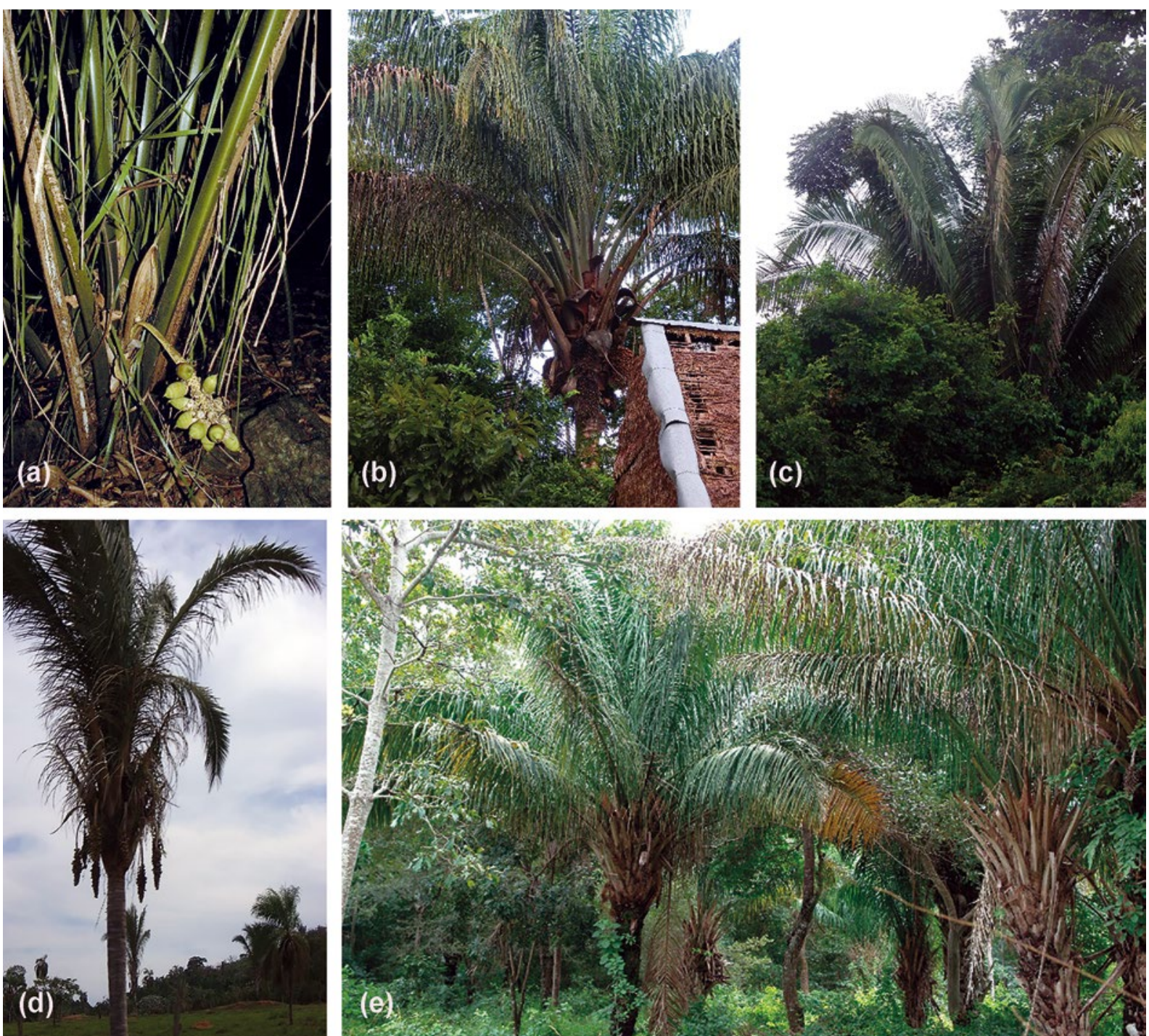

Figura 1. Especies de Attalea y variación morfológica del género en Bolivia. (a) A. eichleri, (b) A. maripa, (c) $A$. peruviana, (d) A. speciosa y (e) A. princeps.

En cada espécimen se realizó la verificación y limpieza de datos de las coordenadas geográficas, en función a la información de campo registrada y en casos problemáticos (como insuficientes datos) al conocimiento previo de la distribución de especies. Para ello se utilizó diferentes mapas temáticos en formato shapefile de provincias, municipios, poblaciones, ríos y en base a otras referencias de coordenadas adyacentes e información cartográfica disponible a partir del Centro Digital de Recursos Naturales de Bolivia (CDRNB, www.cdrnbolivia.org) y GeoBolivia (www. geo.gob.bo/). Toda la base de datos de los especímenes de Attalea fue organizada y proyectada en el programa ARCGIS 10.3.1 (ESRI 2015).

Con la información generada y normalizada (especies vs. sistemas ecológicos), realizamos una clasificación jerárquica de agrupamiento o dendrograma utilizando el método de la distancia mínima (nearest neighbour o single linkage), para ello empleamos el programa R (3.3.2) y el paquete Vegan (Oksanen et al. 2007). Este método procede de acuerdo con el algoritmo general, considerando la distancia entre grupos (clusters) como la distancia mínima entre los individuos más próximos; por lo tanto es espacio-contractivo, es decir tiene a aproximar los indi- viduos más de lo que indicarían sus disimilaridades o distancias iniciales, que ha sido reivindicado por Jardine y Sibson (1971) como matemáticamente preferible por sus propiedades. Este análisis fue refrendado con la información que tenemos de las especies y su presencia en campo.

\section{Resultados}

Las especies de Attalea de Bolivia ocupan una amplia extensión del país y se encuentran asociadas principalmente a zonas boscosas y secundariamente a zonas de paisajes sabanas y pantanos. Además este género está generalmente más asociado a tierras bajas que a formaciones de montańas y serranías (andinas o del Cerrado). La presencia de este género de palmeras se relaciona con 30 sistemas ecológicos que no solo se encuentran en Bolivia, sino también en Perú y por ejemplo $A$. princeps es la que se encuentra en mayor número de sistemas: 17 (57\% del total), mientras algunas especies se asocian en forma exclusiva a determinados sistemas ecológicos, como $A$. blepharopus, $A$. moorei (Glassman) Zona y A. pacencis (Tabla 2). El punto más septentrional de distribución se sitúa apenas más al sur del paralelo $18^{\circ}$ en localidades del departamento de Santa Cruz. El sistema ecológico con mayor número de especies corresponde 
Tabla 2. Puntos de colecciones herborizadas de las especies de Attalea en los sistemas ecológicos de Bolivia. La leyenda de los sistemas ecológicos se detalla en la Figura 2. Leyenda: Sist Ecol. = Sistemas ecológicos (la descripción de las siglas se detallan en la Figura 3), At. bas = Attalea bassleriana, At. ble = Attalea blepharopus, At. eic $=$ Attalea eichleri, At. mar $=$ Attalea maripa, At. moo $=$ Attalea moorei, At. pac $=$ Attalea pacensis, At. per $=$ Attalea peruviana, At. pha $=$ Attalea phalerata, At. pri $=$ Attalea princeps, At. spe = Attalea speciosa, At. tes $=$ Attalea tessmannii.

\begin{tabular}{|c|c|c|c|c|c|c|c|c|c|c|c|c|c|}
\hline Sist. Ecol. & At_bas & At_ble & At_eic & At_mar & At_moo & At_pac & At_per & At_pha & At_pri & At_spe & At_tes & Presencia & $\%$ \\
\hline CES408_570 & 1 & 1 & & & & 1 & 1 & & 7 & & & 5 & 45.5 \\
\hline CES406_231 & & & & 1 & & & & 1 & 2 & 2 & & 4 & 36.4 \\
\hline CES408_518 & 1 & & & 1 & & & 1 & & & 2 & & 4 & 36.4 \\
\hline CES408_531 & 2 & & & 1 & & & & 1 & 1 & & & 4 & 36.4 \\
\hline CES408_544 & & & & 1 & 2 & & 2 & & & & 2 & 4 & 36.4 \\
\hline CES408_578 & & & & 1 & 1 & & & 1 & & 1 & & 4 & 36.4 \\
\hline Co05Beni & & 5 & & 1 & & & & & 5 & 1 & & 4 & 36.4 \\
\hline Antrópico & & & & & & 4 & 1 & 3 & 1 & & & 4 & 36.4 \\
\hline CES406_238 & & & & & & & & 3 & 2 & 1 & & 3 & 27.3 \\
\hline CES406_241 & & & & & & & & 1 & 1 & 1 & & 3 & 27.3 \\
\hline CES408_560 & 1 & & & 1 & & & 1 & & & & & 3 & 27.3 \\
\hline CES408_574 & & & & & & & & & 1 & 1 & 1 & 3 & 27.3 \\
\hline CES406_235 & & & 1 & & & & & & & 1 & & 2 & 18.2 \\
\hline CES406_240 & & & 1 & & & & & 2 & & & & 2 & 18.2 \\
\hline CES408_545 & 1 & & & & & & & & 2 & & & 2 & 18.2 \\
\hline Co02Beni & & & & & & & & 1 & 4 & & & 2 & 18.2 \\
\hline CES406_233 & & & & & & & & 1 & & & & 1 & 9.1 \\
\hline CES406_250 & & & & & & & & & & 1 & & 1 & 9.1 \\
\hline CES406_253 & & & & & & & & & 2 & & & 1 & 9.1 \\
\hline CES406_494 & & & 1 & & & & & & & & & 1 & 9.1 \\
\hline CES408_528 & & & & & & & & & 1 & & & 1 & 9.1 \\
\hline CES408_535 & & & & & & & & & & & 1 & 1 & 9.1 \\
\hline CES408_543 & & & & & & & & & 4 & & & 1 & 9.1 \\
\hline CES408_552 & & & & & & & 2 & & & & & 1 & 9.1 \\
\hline CES408_573 & & & & & & & 1 & & & & & 1 & 9.1 \\
\hline CES409_048 & & & & & & & & & 1 & & & 1 & 9.1 \\
\hline CES409_053 & & & & & & & & & 1 & & & 1 & 9.1 \\
\hline CES409_054 & & & & & & & & & 1 & & & 1 & 9.1 \\
\hline Co01Beni & & & & & & & & & 2 & & & 1 & 9.1 \\
\hline Co06Beni & & & & 1 & & & & & & & & 1 & 9.1 \\
\hline Presencia & 5 & 2 & 3 & 8 & 2 & 2 & 7 & 9 & 17 & 9 & 3 & & \\
\hline$\%$ & 16.67 & 6.67 & 10.00 & 26.67 & 6.67 & 6.67 & 23.33 & 30.00 & 56.67 & 30.00 & 10.00 & & \\
\hline
\end{tabular}

a los bosques de glacis preandino del SWA con cinco especies $(45.5 \%)$, mientras que son siete los que tienen cuatro $(36.4 \%)$ : Bosques chiquitanos de transición a la Amazonia sobre suelos bien drenados, bosque de palmas del centro-sur de la Amazonia, bosque inundable de la llanura aluvial de ríos de aguas blancas del SWA, bosque siempreverde estacional de la penillanura del SWA, bosque inundado por aguas blancas estancadas del SWA, complejo de bosques y vegetación riparia de aguas blancas del Beni y antrópico (Tabla 2).

La presencia de las especies del género Attalea con sistemas ecológicos se detalla a continuación (respecto a las figuras 3-4, según sea el caso):
- Attalea bassleriana (Burret) Zona (Fig. 3), se encuentra mayormente asociada a cinco formaciones de bosque: bosque de palmas del centro-sur de la Amazonia, bosque inundable de la llanura aluvial de ríos de aguas blancas del SWA, bosque siempreverde estacional subandino, sabanas arboladas y arbustivas sobre suelos anegables y bosques del glacis preandino del SW amazónico. Solo representada en el norte del departamento de La Paz.

- Attalea blepharopus Mart. (Fig. 3), que es endémica de Bolivia, está mayormente asociada a complejo de bosques y vegetación riparia, como bosques del glacis preandino del SWA de aguas blancas y complejo de bosques y ve- 


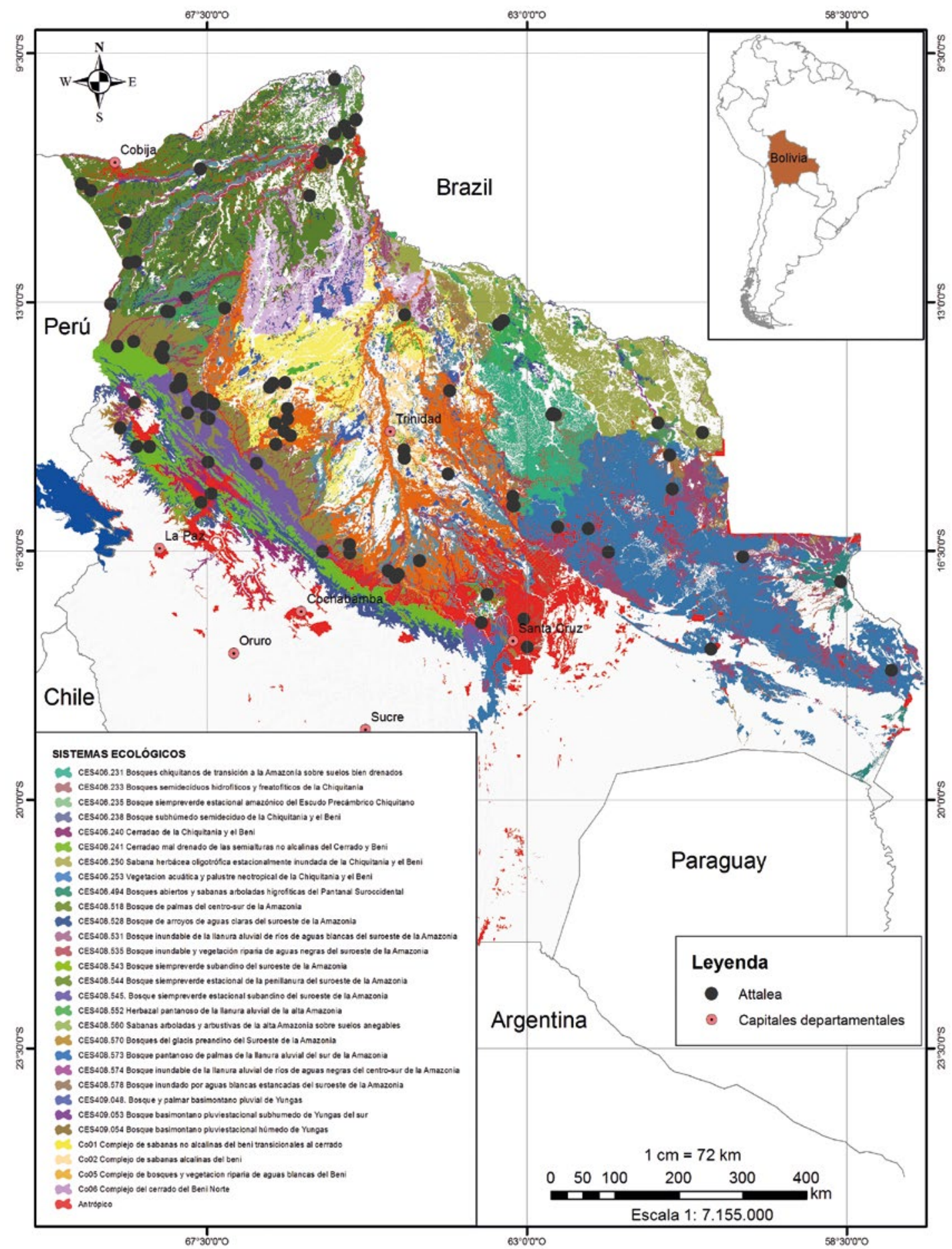

Figura 2. Presencia del género Attalea en relación a sistemas ecológicos de Bolivia según el sistema de Navarro \& Ferreira (2011) y a nivel regional de acuerdo a Young (2007). Los puntos negros corresponden a las colecciones de las 11 especies de Attalea.

getación riparia de aguas blancas del Beni. Compartida entre Cochabamba, Beni y La Paz.

- Attalea eichleri (Drude) A.J. Hend. (Fig. 3), se encuentra asociada a dos formaciones típicas del este de Santa Cruz, bosques siempreverde estacional amazónico del Escudo Precámbrico Chiquitano, formación cerradao de la Chiquitanía y el Beni.

- Attalea maripa (Aubl.) Mart. (Fig. 3), que cubre una amplia distribución geográfica e incluye varias forma- ciones y paisajes de bosque y sabanas, como bosques chiquitanos en suelos bien drenados, bosque de palmas del centro-sur de la Amazonia, bosque inundable de la llanura aluvial amazónica de ríos de aguas blancas del SWA, bosque siempreverde estacional de la penillanura del SWA, sabanas arboladas y arbustivas con suelos anegables, bosque inundado por aguas blancas estancadas del SWA, complejo de bosques y vegetación riparia de aguas blancas del Beni. Se encuentra en los departamentos de Santa Cruz, Beni, norte de La Paz y Pando. 


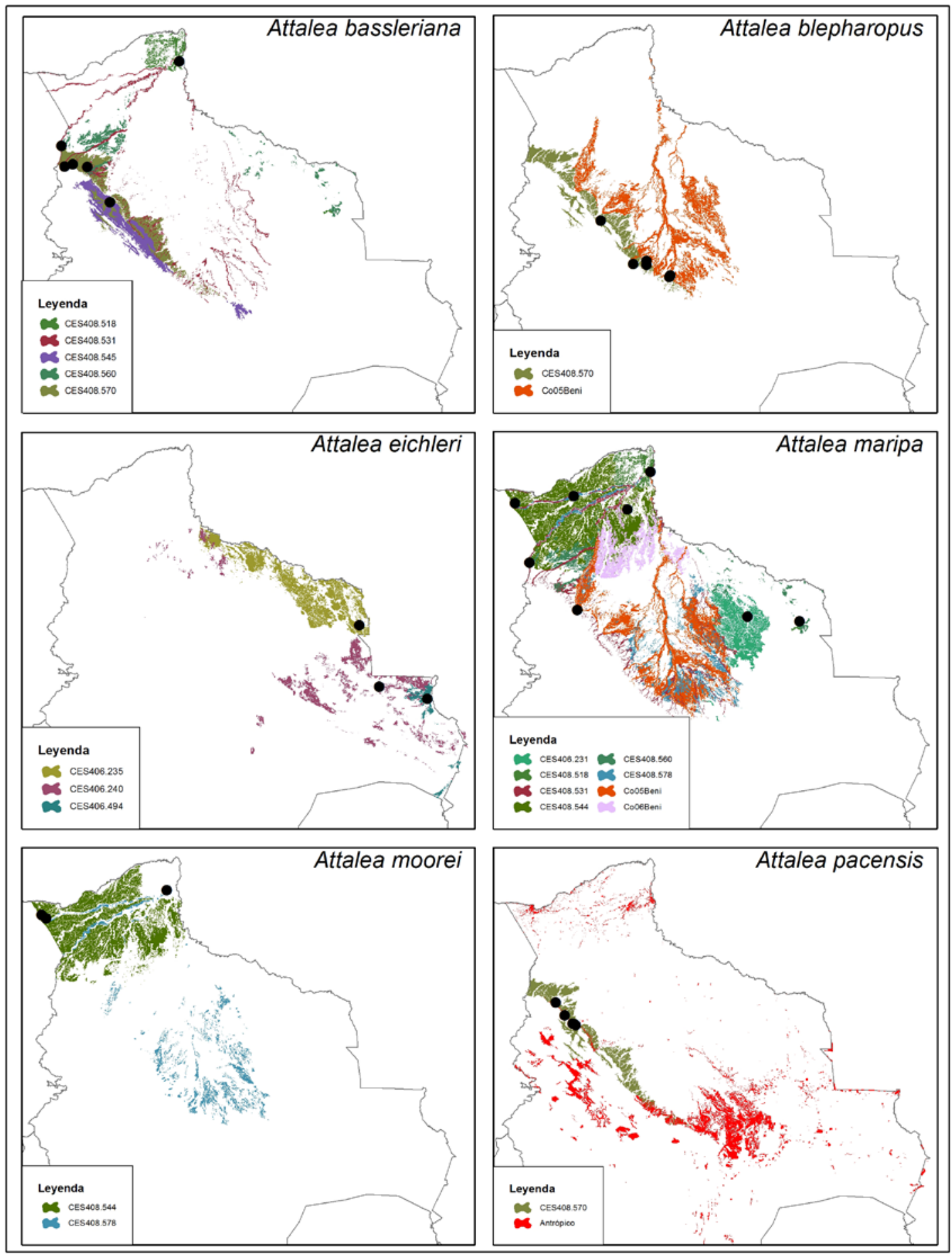

Figura 3. Distribución de A. bassleriana, A. blepharopus, A. eichleri, A. maripa, A. moorei y A. pacensis en Bolivia. La leyenda de los sistemas ecológicos se detalla en la figura 2.

- Attalea moorei (Glassman) Zona (Fig. 3), la cual solamente se encuentra en el departamento de Pando en bosque siempreverde estacional de la penillanura y bosque inundado por aguas blancas estancadas del SWA.

- Attalea pacensis Moraes y Pintaud (Fig. 3), que a la fecha solo ha sido registrada en el norte departamento de La Paz y está asociada con los bosques del glacis preandino del SWA y en formaciones antrópicas.

- Attalea peruviana Zona (Fig. 4), la cual se relaciona con bosque de palmas del centro-sur de la Amazonia, bosque siempreverde estacional de la penillanura y subandino del SWA, herbazal pantanoso de la llanura aluvial amazónica, sabanas arboladas y arbustivas amazónicas sobre suelos anegables, bosques del glacis preandino y bosque pantanoso de la llanura aluvial del SWA. Está representada en los departamentos de La Paz, Beni y Pando.

- Attalea phalerata Mart. ex Spreng. (Fig. 4), está mayormente asociada a formaciones riparias e inundables, como en bosques chiquitanos de transición de la Amazonia sobre suelos bien drenados, bosques semideciduos hidrofíticos 


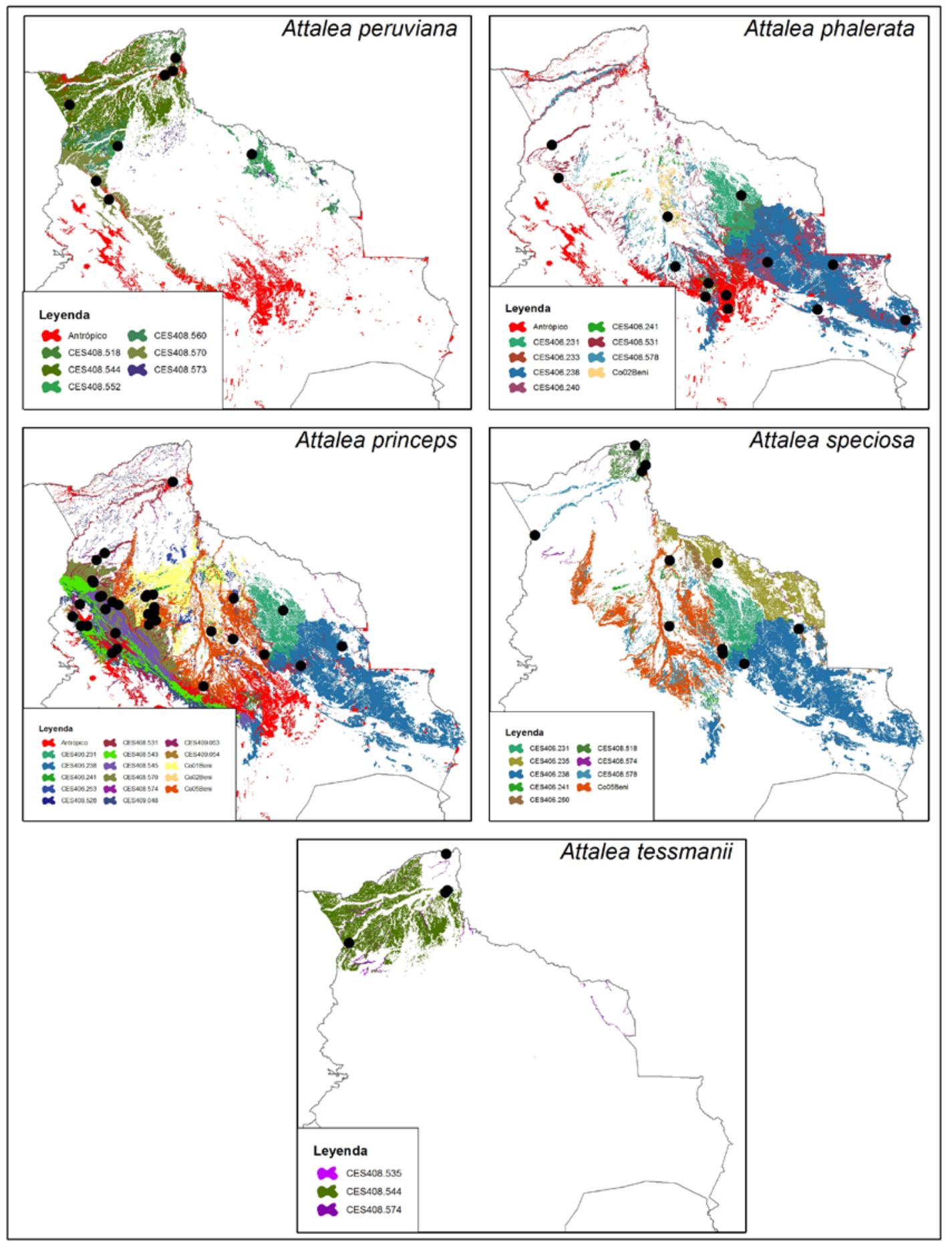

Figura 4. Distribución de A. peruviana, A. phalerata, A. princeps, A. speciosa y A. tessmannii en Bolivia. La leyenda de los sistemas ecológicos se detalla en la figura 2.

y freatofíticos de la Chiquitanía, bosques subhúmedos semideciduo de la Chiquitanía y el Beni, formación del cerradao de la Chiquitanía y el Beni, formación del cerradao mal drenado de las semialturas no alcalinas del Cerrado y Beni, bosque inundable de la llanura aluvial de ríos de aguas blancas del SWA, bosque inundado por aguas blancas estancadas del SWA y complejo de sabanas alcalinas del Beni.

- Attalea princeps Mart. (Fig. 4), ocurre en zonas aluviales y submontanas andinas, como bosque chiquitano de transición a la Amazonia sobre suelos bien drenados, bosque subhúmedo semideciduo de la Chiquitanía y el Beni, Cerradao mal drenado de las semialturas no alcalinas del Cerrado y Beni, vegetación acuática y palustre neotropical de la Chiquitanía y el Beni, bosques del SWA: en arroyos de aguas claras, bosque inundable de la llanura aluvial de ríos de aguas blancas, bosque siempreverde estacional subandino y bosque del glacis preandino, bosque inundable de la llanura aluvial de ríos de aguas negras del centro-sur amazónico, bosque 
y palmar basimontano pluvial de Yungas, bosques basimontano pluviestacional subhúmedo y húmedo de Yungas del sur, complejo de sabanas no alcalinas del Beni transicionales al Cerrado, complejo de sabanas alcalinas del Beni y complejo de bosques y vegetación riparia de aguas blancas del Beni. Se encuentra en Beni, Cochabamba, La Paz, Pando y Santa Cruz.

- Attalea speciosa Mart. (Fig. 4), que se encuentra en bosque y áreas abiertas, como bosques chiquitanos de transición a la Amazonia sobre suelos bien drenados, bosque siempreverde estacional amazónico del Escudo Precámbrico Chiquitano, bosque subhúmedo semideciduo de la Chiquitanía y el Beni, formación del cerradao mal drenado de las semialturas no alcalinas del Cerrado y Beni, sabana herbácea oligotrófica estacionalmente inundada de la Chiquitania y el Beni, bosque de palmas y bosque inundable de la llanura aluvial de ríos de aguas negras del centro-sur de la Amazonia, bosque inundado por aguas blancas estancadas del SWA y complejo del cerrado del Beni norte. Esta especie ocurre en Santa Cruz y Beni.

- Attalea tessmannii Burret (Fig. 4), se encuentra asociado a bosque inundable y vegetación riparia de aguas negras de SWA, bosque siempreverde estacional de la penillanura del SWA y bosque inundable de la llanura aluvial de ríos de aguas negras del centro-sur Amazonia. Es característica de Beni, La Paz y Pando.

Análisis de agrupamiento jerárquico de las especies de Attalea con los sistemas ecológicos.- Como resultado del análisis de agrupamiento jerárquico, se presenta el dendrograma que relaciona las especies de Attalea con los sistemas ecológicos de Bolivia (Fig. 5). La especie más distante o basal resulta siendo A. eichleri - representado en formaciones de sabanas y serranías del Cerrado del este de Bolivia - y que se separa del resto que representa a las especies del SWA. En el grupo de las especies del SWA, el primer grupo con una especie - A. blepharopus presenta su disimilitud respecto a otro grupo conformado por las nueve especies de Attalea. A continuación $A$. princeps y $A$. pacencis - que se encuentran al mismo nivel y que inclusive comparten algunos sistemas ecológicos - presentan igual relación con el grupo conformado por las siguientes siete especies.

Posteriormente se tiene a dos grupos similares, uno conformado por $A$. moorei y $A$. tessmannii relacionados a sistemas amazónicos del norte de Bolivia, mientras que el otro está compuesto por cinco especies. De este segundo grupo se desprenden nuevamente dos grupos: uno conformado por $A$. phalerata muy aparte y aislada, mayormente relacionada con sistemas del Pantanal y la Chiquitanía, mientras que el otro grupo incluye a cuatro especies, siendo el primero formado por $A$. bassleriana y $A$. peruviana que colonizan sistemas aluviales y subandinos y el segundo - con $A$. maripa y $A$. speciosa - relacionados con sistemas chiquitanos, precámbricos y amazónicos.

\section{Discusión}

La representación de la distribución geográfica del género Attalea en Bolivia y su relación con sistemas ecológicos a nivel regional muestra su asociación que facilita la indicación de presencia y que no es exclusiva a nivel de especies. Considerando que las especies de Attalea son fácilmente reconocibles por su talla

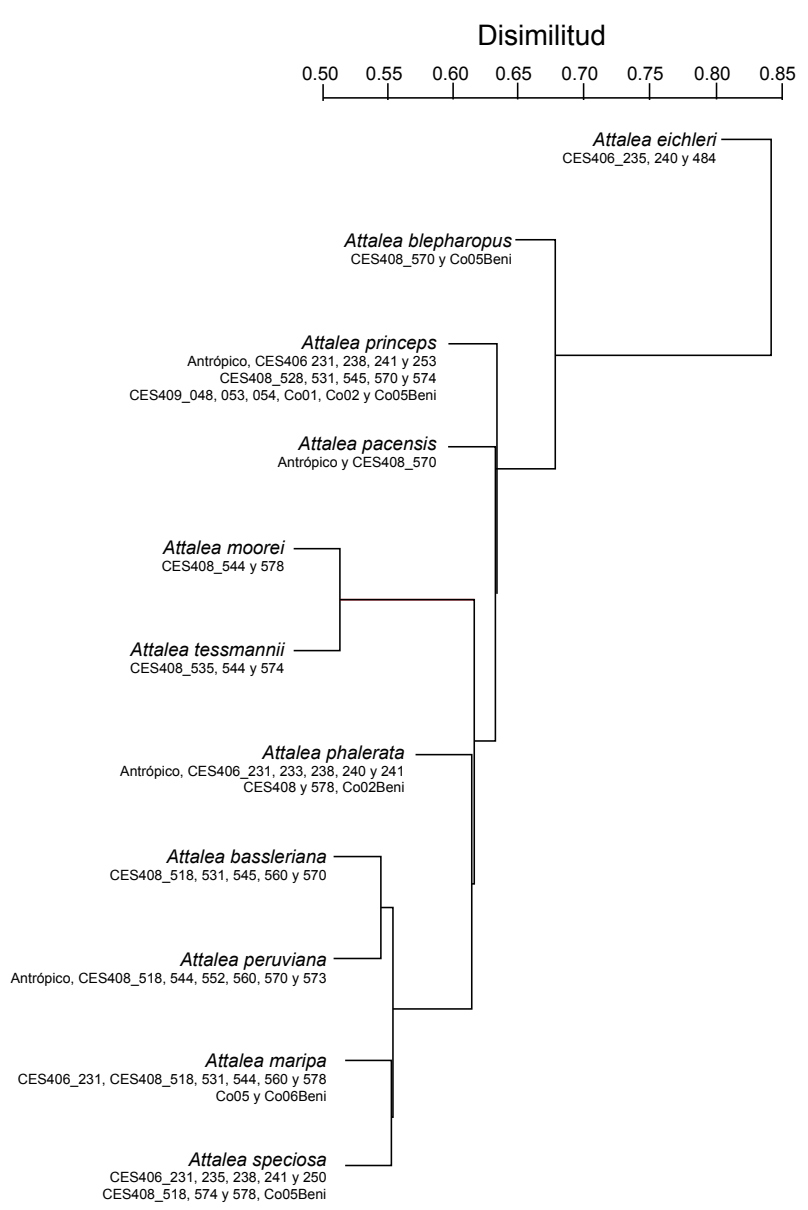

Figura 5. Dendrograma que relaciona por disimilitud a las especies de Attalea de Bolivia con los sistemas ecológicos a nivel regional. Attalea eichleri no se agrupa con el resto de las especies del SWA, ya que procede de formaciones ecológicas del Escudo Precámbrico en la provincia fitogeográfica del Cerrado (este de Bolivia).

y en varios casos por su densidad poblacional (Pintaud 2008), esta evaluación resulta útil para resaltar la presencia de estas especies en relación a los 30 sistemas ecológicos en que fueron reportadas. Precisamente por su talla mayor ( $8-22 \mathrm{~m}$ de altura) la representación en colecciones de herbarios podrá desafiar que se incrementen los registros botánicos en campo y se documente estas evaluaciones a mayor detalle a nivel de la región.

Aunque efectivamente la presencia de la colección de una o más especies en relación a uno de los sistemas ecológicos también pueda ser un artefacto porque no se basa en relevamientos a detalle en campo, en realidad es una aproximación documentada. Sin embargo, en la rectificación de algunos puntos ha tomado en cuenta las formaciones continuas de sistemas, en lugar por ejemplo de seleccionar los sitios antrópicos. Esto favorece la relación de la vegetación potencial y así del sistema ecológico en relación a los puntos de colecciones botánicas, como también fue analizado para todas las especies nativas de la familia Arecaceae de Bolivia por Moraes et al. (2014).

Por otro lado, es importante notar que varias comunidades locales presentan aún individuos que alcanzaron la edad de floración y aquellos que llevan muchos años desde que son reproductivos en sistemas deforestados y que actualmente son relictos o bosques secundarios y hasta sabanas, por ejemplo 
los casos de $A$. princeps y $A$. speciosa, respectivamente. Como fue planteado en Portela et al. (2014) mediante la selección de determinadas semillas o características de especies de palmeras que son aprovechadas para varios fines y aplicaciones generando escenarios reconocidos como antrópicos o modificados.

Según el mapa propuesto para Bolivia y Perú (Young 2007), de los 30 sistemas ecológicos en que se ha reportado la presencia de especies de Attalea en Bolivia, 15 (50\%) están representados en Perú y se circunscriben mayormente a sistemas de la SWA. No se cuenta con una evaluación que haya seguido el mismo sistema de clasificación de esos sistemas ecológicos del SWA representado en Brasil sino a una escala mayor en relación a especies afines con los Andes, formaciones sobre arena blanca (campina, campinarana, sabanas) y formaciones en regiones más secas (matas secas, bosques semi-caducifolios, sabanas) según Dinerstein et al. (1995). Sin embargo, es importante considerar el determinante impacto desde los años 70’s en la región occidental brasileña (por ejemplo la zona del Acre) que se encuentra muy afectada por la deforestación, el desarrollo ganadero y la construcción de carreteras bajo megaproyectos (Hoelle 2014). Por ello, se esperaría un porcentaje de poblaciones relictuales en formaciones de bosques y que son características en sistemas ecológicos del SWA; como por ejemplo Attalea tessmannii es conocida sólo en la cuenca del alto Juruá y fue encontrada por botánicos por segunda vez sólo en 1992 (Silveira \& Daly 1999).

Aunque otros autores no evaluaron el mismo grupo de especies ni su relación con sistemas ecológicos, los resultados obtenidos en este análisis de agrupación de especies de Attalea en Bolivia genera las siguientes implicaciones: 1) El claro distanciamiento de $A$. eichleri del resto de las especies del SWA; que probablemente se deba a que se encuentra en paisajes precámbricos muy antiguos y por ello sea la especie más externa de todas. Esto coincide con el análisis fitogeográfico por Velásquez-Pinto y Bacon (2016) que la sitúa en la provincia fitogeográfica del Cerrado (del centro de Brasil). Aunque Pintaud et al. (2008) mencionaron que son dos especies de amplia distribución en la periferia sur-oeste, sur y sur-este de la Amazonia: Attalea eichleri y A. speciosa, entre otras. 2) Por otro lado, Attalea blepharopus queda primariamente apartada del resto del grupo de especies de Attalea que conforman un agrupamiento jerárquico mayor como palmeras incluidas en el SWA. Se conoce que esta especie es endémica de Bolivia y está asociada generalmente con bosques ribereños del centro del país y que recientemente fue recolectada y epitifipicada después de más de un siglo de su descripción original (Moraes \& Pintaud 2016a) y que se planteó podría estar más emparentada por su morfología con $A$. bassleriana, según Pintaud et al. (2016).

Además en el grupo de las palmeras del SWA, claramente A. phalerata también está aislada entre grupos de especies pues aunque también se la relaciona con bosques y sabanas inundables, se la encuentra en densas poblaciones de la Chiquitanía y especialmente en el Pantanal de Bolivia. La separación de esta especie del resto de los grupos jerárquicos corrobora los hallazgos de otros autores en la filogenie de Attalea como Meerow et al. (2009) y Freitas et al. (2016), aunque según estos segundos autores, el área ancestral de esta especie se ha mantenido en la Amazonia; considerada periférica a la Amazonia pero también presente en formaciones típicamente amazónicas así como bosques de galería y otros lugares húmedos del Cerrado (Pintaud et al. 2008) y en un ámbito muy amplio en el SWA (Pintaud et al. 2016). A su vez, está estrechamente relacionada con los dos grupos: $A$. moorei y $A$. tessmannii que ocupan típicos bosques amazónicos, así como el segundo grupo subdividido entre $A$. bassleriana y $A$. peruviana en sistemas típicamente de bosques amazónicos, así como $A$. maripa y $A$. speciosa, cuya presencia es mucho más variada y amplia para la primera, mientras que la segunda se asocia a bosques amazónicos y áreas abiertas del Cerrado. Precisamente Freitas et al. (2016) mencionaron que uno de los ancestros de $A$. speciosa divergió de un linaje amazónico hace seis millones de años, mientras que $A$. maripa es considerada pan-amazónica (Pintaud et al. 2008).

Rodríguez del Castillo et al. (2016) consideran al complejo phalerata (o clado Scheelea) - conformado por A. phalerata, $A$. huebneri, $A$. weberbaueri, $A$. anisitsiana y hasta 10 especies más, aunque de acuerdo a Pintaud et al. (2016) solo serían siete ( $A$. phalerata, A. princeps, A. moorei, A. weberbaueri, A. anisitsiana, A. pacensis, $A$. huebneri) - que ocupa las partes más húmedas de la Amazonia occidental, incluyendo a Brasil, Perú y Colombia, donde crecen en bosques de várzea y a menudo junto a $A$. bassleriana. En Bolivia este complejo está representado por tres especies: $A$. phalerata, $A$. princeps y $A$. moorei. Según Pintaud et al. (2008), Attalea princeps correspondería al grupo de especies de palmeras asociado a la periferia sur de la Amazonia.

Según Freitas et al. (2016), al considerar las áreas ancestrales reconstruidas en el continente, se reconoce como faja de influencia del Cerrado y de la Amazonia a $A$. speciosa, que junto a A. eichleri componen el clado Orbignya (Meerow et al. 2009), que tuvo un ancestro exclusivamente amazónico que luego se diversificó en formaciones del Cerrado (Freitas et al. 2016). Finalmente el clado Attalea sensu stricto no está representado en Bolivia. De tal forma, que el análisis del presente estudio coincide en que $A$. maripa, $A$. eichleri y $A$. speciosa están registradas en sistemas ecológicos de la Chiquitanía, Cerrado y Amazonia como área ancestral según Freitas et al. (2016). La procedencia ancestral de $A$. maripa, con una amplia distribución actual en la Amazonia, se localizó desde el noroeste de Sudamérica, en valles del Chocó de Colombia con diversificaciones hacia los Andes y la Amazonia (Freitas et al. 2016).

Para el contexto regional y según Pintaud et al. (2016), exceptuando $A$. eichleri, $A$. blepharopus y $A$. speciosa, las restantes ocho especies de Attalea en Bolivia también están representadas en sistemas ecológicos similares de Perú, mientras que en Brasil está ausente únicamente $A$. blepharopus.

Este trabajo contribuye a nivel de la asociación de especies de 11 especies nativas de Attalea en 30 sistemas ecológicos representados en Bolivia y en su relación con uno a cinco sistemas por cada especie. El análisis sobre los grupos de especies asociados con los sistemas ecológicos puede tener implicaciones en la elaboración de un plan de conservación nacional para el grupo, considerando que no todas las especies tienen amplio de distribución y más bien se restringen a determinados tipos de sistemas ecológicos.

\section{Agradecimientos}

Va nuestro agradecimiento a los herbarios USZ y BOLV por proporcionar la información de las colecciones depositadas que fueron parte del análisis que presentamos. A la Dra. Carla Maldonado por sus comentarios referidos al dendrograma de 
Attalea y los sistemas ecológicos. También expresamos nuestra gratitud a los revisores de la revista cuyas observaciones y sugerencias mejoraron el contenido de este trabajo.

\section{Literatura citada}

Dinerstein E., D.M. Olson, D.J. Grahan, A.L. Webster, S.A. Primm, M.P. Bookbinder \& G. Ledec. 1995. A conservation assessment of the terrestrial ecoregions of Latin America and the Caribbean. WWF, The World Bank, Washington.

ESRI. 2015. ArcGIS 10.3.1 for Desktop Online Help. Environmental Systems Research Institute, Inc., Redlands, California, USA.

Freitas C., A. Henderson, A.W. Meerow, J.-C. Pintaud, C.E. Barbosa, L. Noblick, F.R.C. Costa \& D. Barrington. 2016. Phylogenetic analysis of Attalea (Arecaceae): insights into the historical biogeography of a recently diversified Neotropical plant group. Botanical Journal of the Linnean Society 182: 287-302. DOI: http://dx.doi.org/10.1111/boj.12466

Henderson A., G. Galeano \& R. Bernal. 1995. Field guide to the palms of the Americas. Princeton University Press, New Jersey.

Hoelle J. 2014. Cattle culture in the Brazilian Amazon. Human Organization 73(4): 363-374.

Jardine N. \& R. Sibson. 1971. Mathematical taxonomy. J. Wiley and Sons Ltd, London and New York.

Josse C., F. Cuesta, G. Navarro, V. Barrena, E. Cabrera, E. ChacónMoreno, W. Ferreira, M. Peralvo, J. Saito \& A. Tovar. 2009. Ecosistemas de los Andes del norte y centro. Bolivia, Colombia, Ecuador, Perú y Venezuela. Secretaría General de la Comunidad Andina, Programa Regional ECOBONA, CONDESAN-Proyecto Páramo Andino, Programa BioAndes, EcoCiencia, NatureServe, LTA-UNALM, IAvH, ICAE-ULA, CDC-UNALM, RUMBOL SRL, Lima.

Meerow A.W., L. Noblick, J.W. Borrone, T.L.P. Couvreur, M. MauroHerrera, W.J. Hahn, D.N. Kuhn, K. Nakamura, N.H. Oleas \& R.J. Schnell. 2009. Phylogenetic analysis of seven WRKY genes across the palm subtribe Attaleinae (Arecaceae) identifies Syagrus as sister group of the coconut. PLoS ONE 4(10): e7353. DOI: http://dx.doi.org/10.1371/ journal. pone. 0007353 .

Moraes R., M. 2009. Conocimiento actual de la riqueza de palmeras de Bolivia en un contexto geográfico. Revista Grupo de Apoyo a la Biología 4: 11-16.

Moraes R., M. (ed.). 2014a. Palmeras útiles de Bolivia - Las especies mayormente aprovechadas para diferentes fines y aplicaciones. Herbario Nacional de Bolivia, Universidad Mayor de San Andrés, Plural editores, La Paz.

Moraes R., M. 2015. Actualización de la lista de especies de Arecaceae para Bolivia. Revista de la Sociedad Boliviana de Botánica 5(1): 19-28

Moraes R., M., B. Rios-Uzeda, L.R. Moreno, G. Huanca-Huarachi \& D. Larrea-Alcázar. 2014. Using potential distribution models for patterns of species richness, endemism, and phytogeography of palm species in Bolivia. Tropical Conservation Science Journal 7(1): 45-60. https://doi. org/10.1177/194008291400700109

Moraes R., M. \& J.-C. Pintaud. 2016a. Attalea blepharopus Mart. (Arecaceae) from Bolivia revisited since Martius. Candollea 71: 27-32. DOI https://doi.org/10.15553/c2016v711a6

Moraes R., M. \& J.-C. Pintaud. 2016b. A new species of Attalea from Bolivian lowlands. Palms 60(3): 161-168.
Navarro G. \& W. Ferreira. 2011. Mapa de sistemas ecológicos de Bolivia. Escala 1:250.000, CD Interactivo. Consorcio para el Desarrollo Sostenible de la Ecorregión Andina (CONDESAN), The Nature Conservancy (TNC), NatureServe, Rumbol ltda., Cochabamba.

Noblick L.R., W. Hahn \& M.P. Griffith. 2013. Structural cladistic study of Cocoseae, subtribe Attaleinae (Arecaceae): Evaluating taxonomic limits in Attaleinae and the Neotropical genus Syagrus. Brittonia 65: 232-261. DOI https://doi. org/10.1007/s12228-012-9256-y

Oksanen J., R. Kindt, P. Legendre \& B. O'Hara. 2007. The vegan package: community ecology package. https://cran.r-project. org/web/packages/vegan/index.html

Paniagua Zambrana N. Y. \& M. Moraes R. 2009. Hacia el manejo del motacú (Attalea phalerata, Arecaceae) bajo diferente tipo de cosecha (Riberalta, Depto. Beni, NE Bolivia): Estructura y densidad poblacional. Revista Grupo de Apoyo a la Biología 4: $17-23$.

Pintaud J.-C. 2008. An overview of the taxonomy of Attalea (Arecaceae). Revista Peruana de Biología 15, supl. 1:55-63. DOI http://dx.doi.org/10.15381/rpb.v15i3.2968

Pintaud J.-C., G. Galeano, H. Balslev, R. Bernal, F. Borchsenius, E. Ferreira, J.-J. de Granville, K. Mejía, B. Millán, M. Moraes, L. Noblick, F. W. Stauffer \& F. Kahn. 2008. Las palmeras de América del Sur: diversidad, distribución e historia evolutiva. Revista Peruana de Biología 15: 5-27. DOI. http://dx.doi. org/10.15381/rpb.v15i3.2662

Pintaud J.-C., M. Moraes R. \& G. Galeano. 2015. Palmas de Bolivia, Colombia, Ecuador y Perú. Pp. 270-286. En: Balslev, H., M. J. Macía \& H. Navarrete (eds.) Cosecha de Palmas en el Noroeste de Suramérica: Bases Científicas para su Manejo y Conservación. Pontificia Universidad Católica del Ecuador, Quito.

Pintaud J.-C., A. Rodríguez del Castillo, E.J.L. Ferreira, M. Moraes R. \& K. Mejía. 2016. Towards a revision of Attalea in western Amazonia. Palms 60(2): 57-77.

Portela R. C. Q., M. Guedes Braz, M. Bonesso Sampaio \& M. Moraes R. 2014. Distribuição de palmeiras nas florestas tropicais: fatores determinantes. En: T.R. dos Santos Silva, C. Wallace do Nascimento M., L. C. Lima e Lima \& F. de Assis R. dos Santos (eds.) Memorias XI Congreso Latinoamericano de Botànica (octubre), Salvador. Pp. 562-571.

Rodriguez del Castillo A.M., C. García-Dávila, K. Mejía \& J.-C. Pintaud. 2016. Attalea: insights into the diversity and phylogeny of an intriguing genus. Palms 60(3): 109-124.

Silveira M. \& D. Daly. 1999. Estudos sobre a diversidade florística e arbórea. Relatorio analítico. Universidade Federal do Acre - New York Botanical Garden, Brasilia.

Toledo M., J. Balcázar \& T. Ruíz de Centurión. 1999. Usos de la palmera de cusi (Attalea speciosa Mart. ex Spreng) en Yotaú, Santa Cruz, Bolivia. Revista de la Sociedad Boliviana de Botánica 2(2): 175-182.

Velásquez-Puentes F.J. \& C. D. Bacon. 2016. Una introducción a la estructura filogenética de comunidades: Un caso de estudio en palmas de Bolivia. Ecología en Bolivia 51(2): 126-140.

Young B.E. 2007. Endemic species distributions on the east slope of the Andes in Peru and Bolivia. NatureServe, Arlington, Virginia. 